\title{
Avaliação oftalmológica de um grupo de motoristas profissionais de Campinas, São Paulo
}

\author{
Retrospective evaluation of 100 ophtamologic files \\ of professional drivers in Campinas
}

\begin{abstract}
Lucas Barasnevicius Quagliato', Carla Beatriz Carneiro da Cunha Soares², Marcus Vinícius Carneiro da Cunha Soares ${ }^{3}$, Carla Júlia Segre Faiman ${ }^{4}$
\end{abstract}

\begin{abstract}
Quagliato LB, Soares CBCC, Soares MVCC, Faiman CJS. Avaliação oftalmológica de um grupo de motoristas profissionais de Campinas, São Paulo / Retrospective evaluation of 100 ophtamologic files of professional drivers in Campinas. Rev Med (São Paulo). 2012;92(4):261-6.
\end{abstract}

RESUMO: Os condutores profissionais de veículos automotores necessitam de uma acuidade visual adequada, pois a visão é o elemento mais importante do ato de dirigir. OBJETIVO: avaliar as condições visuais de um grupo de 100 motoristas profissionais de Campinas, SP. MÉTODO: Nesse estudo retrospectivo foram revisados 100 prontuários oftalmológicos de motoristas profissionais em atividade, atendidos na Fundação Dr. João Penido Burnier, em Campinas, SP, Brasil, de 2006 a 2011. RESULTADOS: A média de idade dos profissionais foi de 52,8 anos, sendo todos do sexo masculino. As principais razões que motivaram a consulta foram avaliação periódica $(36,8 \%)$ e queixa de baixa acuidade visual (33,6\%). Relataram comorbidades sistêmicas $44 \%$, sendo as mais prevalentes hipertensão arterial e diabetes Apresentaram 100\% de visão $47 \%$ dos sujeitos e em $69,4 \%$ a visão foi igual ou superior a 0,66 , avaliando-se cada olho separadamente. A acuidade visual foi inferior a 0,5 em um dos olhos em $22,1 \%$ dos motoristas. A afecção oftalmológica mais prevalente foi opacificação do cristalino $(44,2 \%)$ e $16,8 \%$ delas tinham indicação de tratamento cirúrgico da catarata. Glaucoma ocorreu em
$3,1 \%$, pterígeo em $5,2 \%$, pinguécula em $6,3 \%$ e aumento da pressão intraocular em $8,6 \%$. A fundoscopia foi normal em $53,6 \%$ dos pacientes. As principais alterações do fundo de olho foram nervo óptico com escavação suspeita (10,5\%), retinopatia diabética $(8,4 \%)$, doença macular relacionada à idade $(6,3 \%)$, retinopatia hipertensiva $(3,1 \%)$, cicatrizes de corioretinite $(3,1 \%)$, descolamento de retina $(3,1 \%)$, coroidose miópica $(3,1 \%)$ e hemorragia vítrea $(2,1 \%)$. CONCLUSÕES: nesse grupo de motoristas profissionais as deficiências visuais apresentaram uma prevalência muito maior do que seria ideal nessa profissão. Pelo menos $22,1 \%$ dos motoristas não poderiam ser profissionais, por não enxergarem 0,5 em um dos olhos. O número de motoristas que seriam reprovados nas categorias $C, D$ e E possivelmente seria maior se fossem submetidos a uma perícia.

DESCRITORES: Acuidade visual; Condução de veículos/ estatística \& dados numéricos; Transtornos da visão/diagnóstico; Registros médicos.

Trabalho realizado no Instituto Penido Burnier, Campinas, SP.

1. Médico especialista em Medicina do Tráfego pela USP, terceiro ano de especialização em Oftalmologia pelo Instituto Penido Burnier, Campinas, SP.

2. Médica especialista em Medicina do Tráfego pela USP, segundo ano de especialização em Oftalmologia pelo Instituto Penido Burnier, Campinas, SP.

3. Médico especialista em Medicina do Tráfego pela USP.

4. Psicóloga do Departamento de Medicina Legal, Ética Médica e Medicina Social e do Trabalho da Faculdade de Medicina da USP. Doutora em Psicologia Clínica pelo Instituto de Psicologia da USP.

Endereço para correspondência: Lucas Barasnevicius Quagliato. Rua Alfredo Calil, 187, Campinas, SP. CEP: 13101507. 


\begin{abstract}
Vision is the main skill for the driving act and a good visual accuity is mandatory to be a professional driver. Objective: to evaluate the visual conditions in a group of 100 professional drivers of Campinas, SP. Methods: This retrospective study rewied records of 100 active professional drivers whose ophthalmologic examination was performed at Fundação Dr. João Penido Burnier de Campinas, a public and private ophthalmologic clinic, from 2006 to 2011. Results: Driver's mean age was 52.8 years and all were males. They came to ophthalmologic evaluation because of periodic evaluation (36.8\%) and impaired visual accuity $(33.6 \%)$. Systemic comorbidities were presented by $44 \%$, with predominance of diabetes and systemic arterial hypertension. The visual accuity was $100 \%$ in $47 \%$ and equal or higher than 0.66 in $69.4 \%$, being each eye avaluated separately. The visual accuity was less than 0.5 in one eye in $22.1 \%$. The most prevalent ophthalmologic
\end{abstract}

\section{INTRODUÇÃO}

Segundo o Ministério da Saúde ${ }^{1}$, a visão é um dos mais importantes meios de comunicação, sendo notadamente vital para o pleno desenvolvimento pessoal e relacionamento do indivíduo com o meio ambiente circundante.

As pesquisas sobre acuidade visual dos condutores de veículos automotores são de extrema relevância, pois mais de $90 \%$ das informações sensoriais enviadas ao cérebro para o ato de dirigir provem da visão. Para executar a maior parte das manobras necessárias para a condução de um veículo, o condutor deve se basear no sentido da visão $0^{2,3}$.

Portadores de doenças oftalmológicas dirigem com menos segurança e tem alto risco de provocar acidentes. O condutor profissional deve ter uma acuidade visual mínima, que propicie condições de detectar e reagir a obstáculos, pedestres, outros veículos e sinalizações ${ }^{2}$. Essa acuidade deve ser de $66 \%$ em cada olho ou $66 \%$ em um olho e $50 \%$ no outro, com visão binocular mínima de $80 \%$. O campo visual deve ser maior que $120^{\circ} \mathrm{em}$ cada olho e deve distinguir as cores verde, amarelo e vermelho, além de ter visão em baixa luminosidade e apresentar recuperação após o ofuscamento direto.

A visão pode ser comprometida por afecções da córnea, do cristalino, da retina, do nervo e das vias ópticas. Os motoristas portadores de catarata, glaucoma, retinopatia diabética, degeneração macular, entre outras doenças oftalmológicas, envolvemse significativamente mais em acidentes de tráfego quando comparados com motoristas que não apresentam essas afecções ${ }^{4}$. Além disso, as alterações cognitivas visuais que, muitas vezes, inauguram um disease was cristalin opacification (44.2\%), with cataract surgery indication in $16.8 \%$. Glaucoma was identified in $3.1 \%$, pterigium in $5.2 \%$, pinguecula in $6.3 \%$ and increased intraocular pression in $8.6 \%$. Fundoscopy was considered normal in $53.6 \%$ and the main pathologies were suspect optic nerve escavation (10.5\%), diabetic retinopathy $(8.4 \%)$, age related macular disease $(6.3 \%)$, hypertensive retinopathy(3.1\%), miopic choroidosis (3.1) and vitreal bleading (2,1\%). Conclusions: Visual impairment prevalence was higher than expected in this group, and at least $22.1 \%$ would not be professional drivers, because they could not see 0.5 in one eye. If they were submitted to a expert review they would be reproved in C, D and E categories.

KEYWORDS: Visual acuity; Automobile driving/statistics \& numeral data; Vision disorders/diagnosis; Medical redords.

quadro de demência, comprometem a orientação visual e espacial. A saúde ocular deve ser, portanto avaliada como um todo.

O pterígio caracteriza-se pelo crescimento de um tecido fibrovascular neoformado encobrindo a esclera, podendo, em raras ocasiões, comprometer o campo visual pela delaminação da córnea. Sua prevalência varia de $2 \%$ nas regiões polares, até $22,5 \%$ nas regiões equatoriais 5 . Quando a proliferação do tecido fibrovascular nãochega a atingir a córnea é chamado de pinguécula.

O comprometimento do cristalino tem intensidades variáveis, indo de uma leve opacificação, que pouco interfere com a visão, até cegueira completa. A catarata é definida como uma opacificação do cristalino, presente em $0,9 \%$ das pessoas entre $40 \mathrm{e}$ 50 anos, $3,2 \%$ entre os 55 e 59 anos, $9,2 \%$ entre os 60 e 64 anos e 21,9: entre os 65 e 69 anos, 33,4\% entre 70 e 74 anos, $51,1 \%$ dos 75 aos 79 anos e em $90,3 \%$ das pessoas com mais de 80 anos $^{6}$.

O glaucoma se caracteriza a um aumento da PIO e comprometimento do nervo óptico. A maioria dos pacientes não percebe a perda visual,que ocorre quando já houve um comprometimento considerável do nervo óptico. Mesmo que um glaucomatoso tenha acuidade visual satisfatória, a diminuição da visão periférica limita a direção veicular ${ }^{4}$.

A degeneração macular relacionada à idade constitui a principal causa de cegueira legal no mundo ocidental acima dos 50 anos de idade. Ocorre em $18 \%$ dos indivíduos acima dos 65 anos e se caracteriza pela presença de drusas no $\mathrm{FO}^{7}$. Caracterizase por diminuição da acuidade visual, com piora no período noturno ${ }^{4}$.

A retinopatia diabética está presente em $3 \%$ 
da população brasileira, sendo também uma das principais causa de cegueira no mundo ${ }^{4}$. A fundoscopia caracteriza-se pela presença de exsudatos e micro-hemorragias. Sua incidência é de $7,6 \%$ entre os 30 e 69 anos de idade, relacionando-se ao tempo de história do diabetes. Dentre os pacientes de insulina - dependentes há mais de 15 anos, 98\% apresentam retinopatia diabética ${ }^{8}$.

Uma complicação grave da retinopatia diabética proliferativa - onde ocorre a formação de neovasos na retina - é a hemorragia vítrea, caracterizada por na intraocular que pode causar perda acentuada da visão ${ }^{8}$.

A retinopatia hipertensiva caracteriza-se na fundoscopia por hemorragias e alterações vasculares. Acarreta comprometimento visual na vigência de uma crise hipertensiva aguda ${ }^{9}$.

A causa mais freqüente de cicatriz de coriorretinite é a toxoplasmose, causadora de $90 \%$ destes casos. Pode comprometer a visão se localizar-se na mácula, área nobre da visão ${ }^{10}$.

O descolamento da retina, definido como separação da retina sensorial do epitélio pigmentar da retina, pode acarretar cegueira se ocorrer na mácula. O principal descolamento é o regmatogênico, causado por ruptura retiniana geralmente espontânea. Incide em 1:10.000 indivíduos por ano, sendo geralmente unilateral. Cerca de $40 \%$ doa descolamentos de retina ocorre em olhos míopes. Seu tratamento é cirúrgico ${ }^{11}$.

A coroidose miópica caracteriza-se por alteração da coróide que ocorre na alta miopia, cursando com diminuição da acuidade visual ${ }^{12}$.

A incidência crescente dos acidentes de tráfego, muitas vezes envolvendo motoristas profissionais, nos despertou para o estudo do comprometimento da visão nessa população. O estudo foi conduzido num centro oftalmológico de atendimento universal, a Fundação Dr. João Penido Burnier, do Instituto Penido Burnier, situado na cidade de Campinas, a 92 km de São Paulo, centro de uma região metropolitana com 2,8 milhões de habitantes, compreendendo 19 municípios.

\section{OBJETIVOS}

\section{Gerais}

Avaliar retrospectivamente 100 prontuários oftalmológicos de motoristas profissionais que exercem a sua atividade na região metropolitana de Campinas, atendidos no Instituto Penido Burnier, no período de 2006 a 2011.

\section{Específicos}

1. Descrever os achados oftalmológicos.

2. Comparar os achados oftalmológicos com os dos indivíduos da mesma faixa etária da nossa população.

3. Avaliar se estes pacientes apresentam acuidade visual suficiente para serem condutores profissionais.

\section{MÉTODO}

\section{População do estudo}

Esse estudo retrospectivo envolveu a análise de 100 prontuários oftalmológicos da Fundação Dr. João Penido Burnier, de Campinas, SP. A anamnese e a avaliação oftalmológica foram padronizadas e continham as seguintes informações: queixa principal em relação à saúde ocular, idade, sexo, e comorbidades do examinado.

\section{Avaliação oftalmológica}

A avaliação oftalmológica compreendeu acuidade visual (AV) aferida em cada olho separadamente, biomicroscopia ocular (BIO), exame de fundo de olho (FO) e tonometria (PIO) em ambos os olhos. Os exames foram realizados por residentes de oftalmologia dessa Instituição.

\section{Critérios de normalidade da acuidade visual}

Foram considerados aptos para exercer a atividade de motorista profissional aqueles que apresentaram AV igual ou superior a 0,66 (66\%) em cada olho. Foram considerado inaptos os que tiveram AV menor ou igual a 0,5 em um dos olhos.

\section{Critérios de normalidade da biomicroscopia}

Ausência de alterações na córnea, cristalino e câmara anterior.

\section{Critério de normalidade da fundoscopia}

Ausência de alterações do nervo óptico, mácula, retina e vítreo.

\section{Critério de normalidade da pressão intraocular}

PIO abaixo de $21 \mathrm{mmHg}$.

\section{RESULTADOS}

Foram avaliados 100 prontuários, dos quais cinco foram excluídos por não apresentarem infor- 
mações suficientes para essa pesquisa.

Todos os 95 motoristas eram homens, profissionais em atividade. Dirigiam caminhões, táxis e ônibus. Procuraram o serviço sem apresentar queixas visuais $36,8 \%$, para uma avaliação periódica de rotina. Vieram à consulta devido à piora da acuidade visual $33,7 \%$ dos motoristas.

A média de idade foi de 52,8 anos, situando-se $9,4 \%$ na faixa acima dos 70 anos, $25,2 \%$ entre os 60 e 69 anos, $56,8 \%$ entre os 40 e 59 anos e $8,4 \%$ com menos de 40 anos.

Relataram comorbidades sistêmicas $44,2 \%$ dos motoristas, sendo $26,3 \%$ hipertensos, $26,3 \%$ diabéticos e 11,5\% com associação destas duas afecções.
A acuidade visual foi de $100 \%$ em cada olho em $47 \%$ dos pacientes.

Em $69,4 \%$ dos pacientes, a acuidade visual em cada olho foi igual ou maior que $0,66(66 \%)$, considerada suficiente para um motorista profissional.

Em 22,1\% a acuidade visual em um dos olhos foi inferior a $50 \%$, excluindo a aprovação num exame de perícia de direção profissional.

A BIO evidenciou opacificação no cristalino em $44,2 \%$ dos pacientes e catarata com indicação cirúrgica em $16,8 \%$ dos pacientes (visão inferior a 0,66 ). A distribuição da faixa etária dos pacientes com catarata e opacificação do cristalino encontrase na Tabela 1.

Tabela 1. Distribuição dos motoristas segundo a faixa etária e a presença de opacificação do cristalino

\begin{tabular}{l|c|c}
\hline faixa etária & $\mathrm{n}$ - de motoristas \% & opacificação do cristalino \% \\
\hline abaixo dos 40 anos & $8(8,4 \%)$ & $0 \%$ \\
\hline $40-59$ anos & $54(56,8 \%)$ & $33,3 \%$ \\
\hline $60-69$ anos & $24(25,2 \%)$ & $66,6 \%$ \\
\hline acima dos 70 anos & $9(9,4 \%)$ & $88,8 \%$ \\
\hline
\end{tabular}

Apresentavam diagnóstico prévio de glaucoma $3,1 \%$. Pterígio foi observado em $5,1 \%$, pinguécula em $6,3 \%$.

A PIO foi avaliada em 46 pacientes, encontrando-se elevada em quatro $(8,6 \%)$.

A fundoscopia foi normal em $53,6 \%$ dos pacientes. Dentre as alterações observou-se suspeita de alteração na escavação do nervo óptico em 10,5\%, retinopatia diabética em $8,4 \%$, dos quais dois apresentaram hemorragia vítrea. Um dos pacientes com hemorragia vítrea procurou atendimento por apresentar perda grave e aguda da acuidade visual.

Alterações retinianas secundárias à hipertensão arterial ocorreram em 3,1\%, descolamento de retina em $3,1 \%$ e cicatriz de coriorretinite em 3,1\%.

Coroidose miópica foi observada em 3,1\%, doença macular relacionada à idade em 6,3\%, um paciente apresentou neuropatia alcoólica e um degeneração retiniana a esclarecer.

\section{DISCUSSÃO}

Nossa população de estudo reflete um predo- mínio de motoristas profissionais do sexo masculino, comum no nosso meio.

A média de idade foi de 52,8 anos, com ampla distribuição nas diversas faixas etárias, concentrando-se $56,8 \%$ dos motoristas na faixa de 40 aos 59 anos. Situaram-se na faixa etária acima dos 70 anos nove motoristas $(9,4 \%)$, ainda em atividade.

As comorbidades encontradas neste grupo de condutores revelam doenças sistêmicas em $44,2 \%$, podendo estas se relacionar a um comprometimento visual. A incidência de diabetes foi de $26,3 \%$, a de hipertensão arterial foi de $26,3 \%$ e a associação de ambas ocorreu em $11,5 \%$. A incidência de hipertensão arterial não diferiu do esperado para a população (22,4\% a $43 \%$ da população brasileira é hipertensa), enquanto a de diabéticos foi superior ao esperado para a nossa população, onde $7,6 \%$ apresentam esse distúrbio metabólico. Essa tendência a um número mais elevado de motoristas com diabetes reflete a procura por um centro oftalmológico multidisciplinar de referência, devido às manifestações oftalmológicas da doença.

A acuidade visual foi maior que $0,66 \mathrm{em}$ cada 
olho em $69,4 \%$ dos condutores, classificando-os como aptos para as categorias C, D e E. Em 22,1\% deles, a visão em um dos olhos foi inferior a $50 \%$, excluindo a possibilidade de serem aptos para a direção profissional. Não foi realizada uma perícia completa nesse grupo, pois não foi avaliada a visão binocular, campo visual, visão cromática, visão noturna e ofuscamento.

Caso houvesse essa perícia, provavelmente esse número de inaptos seria maior.

A incidência de opacificação do cristalino e catarata no grupo de condutores foi de $44,2 \%$, tendo indicação cirúrgica $16,8 \%$, por apresentarem acuidade visual menor que 0,66 . Em todas as faixas etárias a incidência de opacificação/catarata foi maior do que na população geral. Na faixa 40-59 anos a incidência foi de $33,3 \%$, sendo essa incidência na população geral de $9,2 \%$. Na faixa dos 60-69 anos, a incidência foi de $66,6 \%$, contra $21,9 \%$ na população geral6,13. Acima dos 70 anos, dos nove motoristas, oito apresentavam catarata e um já havia sido operado dessa afecção.

O grande número de portadores de opacificação do cristalino nessa população relaciona-se, possivelmente, aos seus hábitos de vida - fumo, álcool e exposição à luz ultravioleta. A alta incidência de diabetes também pode se relacionar a um aumento de incidência de catarata.

As incidências de pterígio $(5,2 \%)$ e pinguécula $(6,3 \%)$ nos motoristas foram consoantes com as da população geral, apesar desse grupo se expor mais tempo à luz solar e à poeira.

A doença macular relacionada à idade foi observada em $6,3 \%$ da casuística, não havendo dados epidemiológicos na população brasileira. Acima dos 65 anos de idade, a incidência da doença macular é de $17 \%$. Fatores além do envelhecimento como exposição solar, tabagismo e hipertensão arterial também podem se relaciona a essa afecção.

A retinopatia diabética apresentou uma prevalência de $32 \%$ nos 25 motoristas diabéticos, não diferindo da prevalência da população geral de diabéticos, sendo de cerca de 100\% no diabetes tipo 1

\section{REFERÊNCIAS}

1. Brasil. Ministério da Saúde. Brasília; 2005 [citado 23 nov. 2011]. Disponível em: http:/portal.saude.gov.br/portal/saúde/Gestor/visualizar_testo.cfm?idtxt=27720\&janela=1.

2. Adura FE. Medicina de tráfego. São Paulo: Associação Brasileira de Medicina de Tráfego; 2011. cap. 13, p.33.

3. Brandão EO, Oliveira NM, Pereira MVC, Maia NCF, Pereira GC. Acuidade visual de motoristas de veículos e mais de $60 \%$ no tipo 2 .

A PIO estava aumentada em $8,6 \%$ dos pacientes aferidos (total de 46). Esse exame deveria ter sido realizado em todos os pacientes, pois, uma vez detectado o aumento da PIO, devem ser solicitados exames complementares para investigar glaucoma.

As alterações do nervo óptico ocorreram em $10,5 \%$ dos condutores, sendo indicada uma investigação complementar para esclarecimento da sua relevância.

Essa amostra de pacientes não é totalmente representativa da população geral dos motoristas, pois reflete a demanda por atendimento oftalmológico de um centro de referência. Esses dados, no entanto, obtidos num grupo profissional que atua numa atividade em que a visão é extremamente importante, representa a realidade da baixa qualidade da visão dos motoristas profissionais do nosso meio.

\section{CONCLUSÕES}

1. Observou-se um predomínio de motoristas profissionais do sexo masculino, com média de idade de 52,8 anos, concentrando-se $56,8 \%$ na faixa de 40 a 59 anos. Apresentaram comorbidades sistêmicas $44,2 \%$ dos condutores, podendo estas se relacionar a um comprometimento visual.

2. $69,4 \%$ foram considerados aptos para serem motoristas profissionais, do ponto de vista oftalmológico; $22,1 \%$ foram inaptos e $8,5 \%$ necessitaria de uma perícia completa.

3. A incidência de catarata foi maior que a da população em todas as faixas etária, refletindo a ação dos fatores de risco nesse grupo profissional.

4. As patologias observadas na fundoscopia não diferiram da população geral.

5. De cada cinco motoristas em atividade, um não estava apto para dirigir, segundo a avaliação oftalmológica.

pesados numa rodovia de grande circulação: problemática e sugestões. Arq Bras Oftamol. 1995;121(6):121-6.

4. Adura FE. Medicina de tráfego. São Paulo: Associação Brasileira de Medicina de Tráfego; 2011. cap. 14, p. 39.

5. Alves MR, Angotti HS. Pterígio. In: Alves MR. Série oftalmológica brasileira. Rio de Janeiro: Guanabara Koogan; 2008. cap. 46, p. 461. 
Quagliato LB et al. Avaliação oftalmológica de um grupo de motoristas profissionais.

6. Arieta CEL. Cegueira e epidemiologia da catarata senil. In: Alves MR. Série oftalmológica brasileira. Rio de Janeiro: Guanabara Koogan; 2008. cap. 3, p. 23.

7. Isaac DLC, Ávila M. Doenças adquiridas da mácula. In: Alves MR. Série oftalmológica brasileira. Rio de Janeiro: Guanabara Koogan; 2008. cap. 4, p. 113.

8. Gil Al, Lavinsky J. Retinopatia diabética. In: Alves MR. Série oftalmológica brasileira. Rio de Janeiro: Guanabara Koogan; 2008. cap. 7, p. 169.

9. Ávila M, Fernandes RS. Doenças vasculares da retina. In: Alves MR. Série oftalmológica brasileira. Rio de Janeiro: Guanabara Koogan; 2008. cap. 6, p. 165.

10. Ávila M, Cella W. Anormalidades da interface vítreorretiniana. In: Alves MR. Série oftalmológica brasileira.
Rio de Janeiro: Guanabara Koogan; 2008. cap. 5, p. 155.

11. Taleb AC, Bianchi LCSN, Ávila M. Anormalidades retinianas periféricas. In: Alves MR. Série oftalmológica brasileira. Rio de Janeiro: Guanabara Koogan; 2008. cap. 15, p. 362.

12. Lavinsy F, Lavinsky J. Distrofias hereditárias da retina e coróide. In: Alves MR. Série oftalmológica brasileira. Rio de Janeiro: Guanabara Koogan; 2008. cap. 12, p. 295.

13. Ribeiro MRF, Mory DB, Vendramini MF, Giuffrida F, Dib SA, Moisés RS, Chacra AR. Diabetes melito. In: Lopes AC. Tratado de clínica médica. 2a ed. São Paulo: Rocca; 2009. cap. 330, p. 3546. 